\title{
Optical Beam Splitting and Switching Based on Arrays of Tilted Bragg Gratings in Planar Waveguides
}

\author{
Nina Podoliak, Matthew T. Posner, James C. Gates, Peter G. R. Smith, Peter Horak* \\ Optoelectronics Research Centre \\ University of Southampton \\ Southampton SO17 1BJ, United Kingdom \\ *peh@orc.soton.ac.uk
}

\begin{abstract}
We investigate theoretically devices based on arrays of parallel planar waveguides inscribed with tilted Bragg gratings. We demonstrate polarization-dependent $N$ by $N+1$ linear couplers. Deterministic switching can be achieved by coherent control of input beams with optimized apodization of the gratings.
\end{abstract}

Keywords-Bragg gratings; Integrated optics devices; Polarization-selective devices.

\section{INTRODUCTION}

Optical beam splitting and switching are important functionalities for integrated optical systems for applications in communications, sensing and quantum information processing. While traditional approaches are based on co-directional couplers, we are currently investigating an alternative technology exploiting tilted Bragg gratings inscribed in straight waveguides. Such gratings can be fabricated with extremely high precision, for example polarization-splitting with over 30 $\mathrm{dB}$ extinction ratio has been demonstrated in fiber [1], and we have recently shown first integrated devices $[2,3]$.

Here we investigate numerically and analytically the wider potential of this technology. We consider and optimize the apodization of the gratings in order to maximize the coupling of light between parallel waveguides. Furthermore, we allow for three and more waveguides thus creating devices with $N$

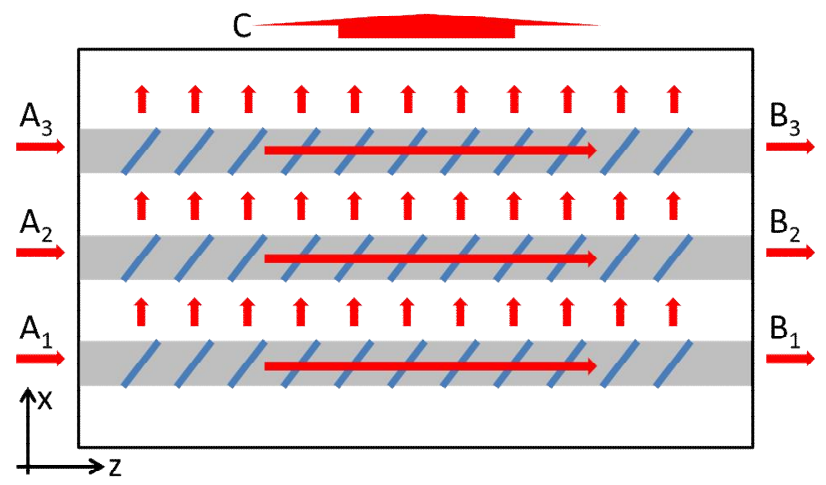

Figure 1. Schematic of investigated devices.

The authors acknowledge financial support from EPSRC through the UK National Quantum Technologies Programme, project EP/M013243/1

"Networked Quantum Information Technologies (NQIT)" and project EP/M013294/1 "UK Quantum Technology Hub for Sensors and Metrology". input and output ports. Finally, we investigate the coherent control of one input beam by a second input beam in another waveguide.

\section{METHODS}

We consider an array of $N$ parallel planar optical waveguides with Bragg gratings inscribed by direct laser writing, as shown in Fig. 1 for $N=3$. The device thus has $N$ input ports $A_{n}$ and output ports $B_{n}$. The gratings are inscribed at a tilt angle of $45^{\circ}$ leading to scattering of light out of the waveguides at $90^{\circ}$ (x-direction). For weak grating strengths this angle corresponds to the Brewster angle and thus only TM polarized light (y-polarization) is scattered, whereas TE polarized light (x-polarization) is transmitted straight from ports $A_{n}$ to $B_{n}$. The scattered TM light can then be scattered back into another waveguide (e.g. from $A_{1}$ into $B_{2}, B_{3}$, etc.) or can exit the device through the side (port $\mathrm{C}$ ).

For our numerical simulations we employ two-dimensional finite element simulations (FEM) with Comsol Multiphysics ${ }^{\circledR}$. We also developed an analytical model of the coupling between the different waveguides following [4].

\section{RESULTS}

In Fig. 2 we show the basic principle of device operation considering a simple device of only two parallel waveguides with spatially uniform gratings. Here light is launched only into waveguide 1 (port $A_{1}$ ). Because of scattering out of the waveguide by the tilted grating, the propagating field amplitude decays exponentially. A fraction of this sidescattered light is then coupled into waveguide 2. Eventually, this light propagating in waveguide 2 also gets scattered sideways, leading to the observed decay in waveguide 2 . Analytically, the electric fields $E_{1}$ and $E_{2}$ in waveguides 1 and 2 , respectively, behave as

$$
\begin{gathered}
E_{1}(z)=E_{0} \exp \{-\alpha z / 2\} \\
E_{2}(z)=\alpha z E_{0} \exp \{-\alpha z / 2\}
\end{gathered}
$$

where $\alpha$ is linked to the device architecture and obtained by generalizing the result of [4]. 

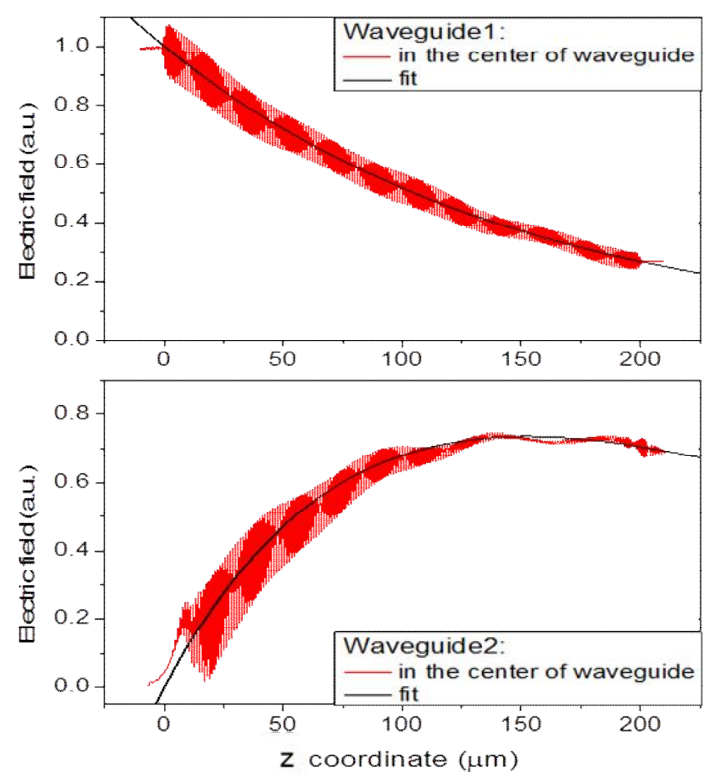

Figure 2. Spatial dependence of the electric field amplitudes in waveguides 1 and 2 for uniform gratings, when light is launched only into input port $A_{1}$. Red curves present numerical FEM data and black curves are obtained using our analytical model.

Further analytical results can be derived in the general case of $N$ waveguides and for arbitrary apodized (z-dependent) grating profiles. In this case, very general linear couplers with $N$ input ports and $N+1$ output ports (including port C) can be designed and optimized, with the only restriction that the tilted gratings can scatter light into the $+x$ and $+z$ directions, i.e., coupling occurs only from waveguides $n$ to $m$ with $m>n$, but not vice versa.

As an example of a device with optimized apodized gratings we investigated the coherent control of light with light. Here we assume a device with $N=2$. Light is launched with the same power into both input ports $A_{1}$ and $A_{2}$ and the relative phase of the two launched light beams is controlled. The apodization of the grating in waveguide 2 is designed such that the light scattered out of waveguides 1 and 2 into the $+\mathrm{x}$ direction interferes destructively at every point in $\mathrm{z}$. Thus, no light exits the device through port $\mathrm{C}$ and all the light emerges from port $B_{2}$ (black curve in Fig. 3). When the phase of the $A_{2}$ input is changed by $\pi$, the opposite is true and all light is emitted from $\mathrm{C}$, and none from $\mathrm{B}_{2}$ (red curve). When

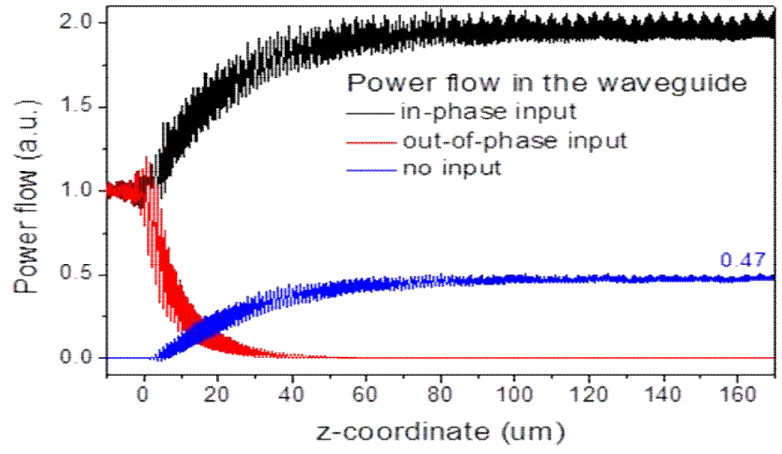

Figure 3. FEM simulation of coherent control of power flow in a 2 waveguide device. The same light power is launched into both waveguides at ports $A_{1}$ and $A_{2}$. The output at port $B_{2}$ is controlled by the phase of input $A_{2}$.

no light is launched into $\mathrm{A}_{2}$, the light scattered from waveguide 1 appears roughly to $50 \%$ in both $\mathrm{B}_{2}$ and $\mathrm{C}$ (blue curve).

\section{CONCLUSIONS}

To summarize, we have investigated a new class of linear planar waveguide devices with $N$ input ports and $N$ discrete plus one distributed output ports. The device consists of $N$ parallel integrated waveguides and coupling between the waveguides is achieved through tilted and apodized Bragg gratings. A wide range of functionalities can be achieved with this geometry with potential applications in optical telecommunications, sensing, and quantum information processing.

\section{REFERENCES}

[1] K. Zhou, G. Simpson, X. Chen, L. Zhang, and I. Bennion, "High extinction ratio in-fiber polarizers based on 45 degree tilted fiber Bragg gratings," Opt. Lett. 30, 1285-7 (2005).

[2] M. T. Posner, P. L. Mennea, N. Podoliak, P. Horak, J. C. Gates, and P G. R. Smith, " $45^{\circ}$ tilted gratings for silica-based integrated polarizers," in European Conference on Lasers and Electro-Optics - European Quantum Electronics Conference (2015), p. CE 105.

[3] M. T. Posner, P. L. Mennea, N. Podoliak, P. Horak, J. C. Gates, and P. G. R. Smith, "Integrated polarizing coupler based on tilted gratings," in OSA Integrated Photonics Research, Silicon, and Nano-Photonics (2016), p. IW3B.3.

[4] T. Yoshino, "Theoretical analysis of a tilted fiber grating polarizer by the beam tracing method," J. Opt. Soc. Am. B, vol. 29, pp. 2478-2483, September 2012 . 